\title{
New approaches to treating resistant depression
}

\author{
Philip J. Cowen \& Ian M. Anderson
}

\begin{abstract}
SUMMARY
Persistent major depression that does not respond to adequate first- or second-line treatment is a common problem in psychiatry. This article updates evidence on recommended treatment strategies and reviews the prospects of more experimental approaches. The main pharmacological development in recent years has been the demonstration that several atypical antipsychotic drugs are effective adjunctive agents in improving symptoms in depression unresponsive to selective serotonin reuptake inhibitors, although their adverse effect burden is high. There is optimism about novel pharmacological strategies based on glutamatergic and anti-inflammatory mechanisms. It is important to combine drug and psychological treatments whenever possible. With persistent therapeutic engagement, the majority of patients remit eventually, but subsequent relapse remains a problem. Clinicians should pursue an active and collaborative treatment plan that makes use of all effective therapeutic modalities and continues into the relapse-prevention phase.
\end{abstract}

\section{LEARNING OBJECTIVES}

- Be aware of the concept of treatment-resistant depression as a staged condition and of the limitations of this concept

- Update knowledge of the efficacy of recommended treatments for resistant depression, including new pharmacological and brain stimulation approaches

- Be aware of the need for persistent therapeutic engagement and time-limited treatment trials to achieve remission and prevent relapse

\section{DECLARATION OF INTEREST}

P.J.C. has been a member of a Lundbeck advisory board. I.M.A. has been a member of advisory boards to Lundbeck, Servier and Alkermes

Many patients with clinical depression recover well with first-line treatment in primary care; such treatment may include psychotherapy and/ or antidepressant drugs, typically a generic selective serotonin reuptake inhibitor (SSRI). In the UK, a relatively small proportion of depressed patients are referred to psychiatrists. A number of factors may underlie these referrals, for example perception of increased risk or psychiatric comorbidity, but often referred patients will have failed to respond satisfactorily to initial attempts at therapy and are therefore considered 'treatment resistant'. An important issue for the clinician at this point is to decide how far further specific pharmacological treatment is indicated and if so, what it should be. A number of evidence-based 'next-step' treatments are available, but at the moment there are only a few clinical pointers, and no established biological markers, to help decide between these options for a particular patient. Consequently, the experience and expertise of patient and clinician play a key role in what should be a supportive therapeutic collaboration (Cowen 2011; Anderson 2013).

Despite the current difficulty in matching individual patients to pharmacological treatment, there is evidence that algorithm-guided approaches are superior to treatment as usual in patients with depression (Bauer 2009). This suggests that a structured approach with explicit 'next steps' if current therapy proves unsuccessful is helpful for both clinician and patient. At each new treatment step, at least some patients are likely to remit (Rush 2006), which shows the value of persistence and optimism in improving relief from depression. Unfortunately, the relapse rates in the year following successful remission increase sharply with the number of treatment trials that have been necessary to achieve it (Rush 2006); this indicates the need for active steps to maintain remission and for further research into optimising longer-term outcomes.

It may be helpful to read this article in conjunction with our earlier reviews in Advances (Anderson 2003; Cowen 2005), which summarised a general approach to the management of resistant depression and the way that pharmacological treatment strategies can be staged and classified. Here we focus on new evidence and approaches in this clinical area.

\section{Definition and assessment}

\section{Definition}

The term 'resistant depression' is typically applied to a depressive disorder for which antidepressant medication (rather than psychotherapy) has failed.
Philip Cowen is a Medical Research Council clinical scientist and Professor of Psychopharmacology at the University of Oxford. He is also Honorary Consultant Psychiatrist at the Oxford Health NHS Foundation Trust. Ian Anderson is Professor of Psychiatry at the University of Manchester and an Honorary Consultant Psychiatrist at Manchester Mental Health and Social Care Trust. Correspondence Professor Philip J. Cowen, University Department of Psychiatry, Warneford Hospital OX3 7JX, UK. Email: phil.cowen@psych. ox.ac.uk 
It has been estimated that about a third of patients with major depression do not respond to treatment with a single antidepressant drug given in adequate dosage for an appropriate period. Around half of these patients will respond if switched to another antidepressant medication (Anderson 2003; Cowen 2005). A further term, 'refractory depression' is sometimes used for patients who fail to improve after multiple treatments, including electroconvulsive therapy (ECT), and whose depression has become chronic (Anderson 2003).

The Sequenced Treatment Alternatives to Relieve Depression $\left(\mathrm{STAR}^{\star} \mathrm{D}\right)$ study, a pragmatic randomised trial of initial and next-step pharmacological treatments of depression, found that after two failed medication trials the likelihood of responding to a subsequent pharmacological treatment becomes significantly lower. Indeed, the chances of response to a new therapy diminish further as the number of failed trials increases (Rush 2006).

The classification of treatment resistance in depression has traditionally been through applying a staging method based on the number of treatments that have been unsuccessfully employed (Cowen 2005). Recent classifications have included other relevant clinical factors. For example, the Maudsley Staging Method derives a score based on severity and duration of the current episode as well as the history of previous treatment (Fekadu 2009). However, the lack of an agreed definition of treatment-resistant depression, the tendency to focus exclusively on drug treatments at the expense of the wider clinical picture and history, the exclusion of psychological treatments, and the message given to the patient have led to a questioning of the clinical usefulness of categorising patients as 'treatment resistant'. Guidance from the National Institute for Health and Care Excellence (NICE), for example, prefers to emphasise treatment-sequencing options rather than identifying a type of patient (NICE 2009).

\section{Assessment}

Whatever one's position about the use of the term treatment resistance, a systematic approach is required when treating a patient not responding to antidepressant medication. First, it is obviously necessary to confirm the diagnosis of depression and to ensure that medication has been taken correctly for a reasonable length of time. A number of clinical factors are known to decrease the likelihood of response to antidepressant medication, including comorbidity (particularly anxiety disorders, substance misuse and physical illness), a history of childhood abuse, ongoing social stresses and lack of social support (Bennabi 2015). Addressing these factors where possible may improve outcome, although clearly not all are amenable to clinical intervention.

Personality factors and interpersonal problems are important in assessing the appropriate treatment for a depressed person. However, it is necessary to distinguish effects of illness from long-standing personality and interpersonal issues: there is a danger of automatically attributing lack of improvement to these latter factors, and subtly blaming the patient. Other important factors in assessment are the presence of depressive psychosis or depression in the context of a bipolar disorder, because in such cases response to single antidepressant treatment is less likely and other pharmacological approaches are more helpful (Cowen 2005). Clinical studies of antidepressant efficacy often distinguish 'response' (a minimum decrease of $50 \%$ on a standard depression rating scale such as the Hamilton Rating Scale for Depression, HRSD) from 'remission', where persisting symptoms are minimal (a score of $\leq 7$ on the HRSD). Remission is self-evidently the better outcome and is now recognised as the goal of treatment where possible, not least because it reduces the risk of subsequent relapse (Cowen 2005).

A key part of the assessment is to obtain a clear description of treatments of all modalities that have already been tried in the current episode. Responses and lack of response in previous episodes are also useful pointers, but should not automatically prevent further prospective trials of treatment. Some patients might not respond to pharmacological treatment because they have experienced severe adverse effects; this needs to be distinguished from lack of response to adequate therapy. In deciding how far to pursue pharmacological treatment, it is helpful to obtain a history of the usual level of functioning and adjustment prior to the depressive episode. Obtaining this history with clarity can be difficult when a person is in the throes of depression and it is generally helpful to review the history with the patient and a family member or close friend. Where a patient has previously functioned well, it is worth pursuing pharmacological treatment until remission is achieved as part of a shared goal, if the patient agrees with this strategy.

\section{Next-step pharmacological treatments}

\section{Switching}

The NICE depression guideline (NICE 2009) recommends that patients who fail to respond to initial SSRI treatment at an optimised dose should be switched to a second SSRI or another newer 
antidepressant drug, for example mirtazapine. Intuitively, it seems that if a patient has not responded to one particular pharmacological approach it would make sense to use a drug with a different mechanism of action for the next treatment trial; however, the evidence for this is weak. For example, a meta-analysis of randomised studies suggested that switching from an SSRI to a different class of antidepressant drug (bupropion, mirtazapine, venlafaxine) rather than a further SSRI was marginally better in terms of remission rate, but the number needed to treat (NNT) was high (22) and of doubtful clinical significance (Papakostas 2008). However, when considering the venlafaxine studies alone, there is a modest advantage in switching to venlafaxine rather than another SSRI in patients who failed respond to initial SSRI treatment $(\mathrm{NNT}=13)$. The tricyclic antidepressants amitriptyline and clomipramine have traditionally been regarded as useful in severe depression and can be considered for individual treatment-resistant patients provided they can be used safely (Cowen 2005).

Monoamine oxidase inhibitors (MAOIs) continue to have a place in the treatment of resistant depression, but they are usually employed at a late stage because of dietary restrictions and drug interactions. Combination of lithium with MAOIs has long been regarded as a particularly helpful strategy (Cowen 2005). Whether MAOI treatment will continue to be an option in the future is uncertain as new generations of practitioners become less familiar with their use. In addition, perhaps because they are becoming 'niche' products, the cost of some MAOI treatments may become unsupportable. For example, although the current price of generic phenelzine remains comparatively low in the UK, generic tranylcypromine is now about $£ 8$ per $10 \mathrm{mg}$ tablet, which means that to treat a patient with a dose of $30 \mathrm{mg}$ a day for a year would cost almost $£ 9000$.

\section{Combination and augmentation strategies}

Adding a second agent to a primary antidepressant tends be called 'combination treatment' if the second drug is considered an antidepressant in its own right and 'augmentation' if it is not. Combination treatments are popular in patients with resistant depression and are usually employed when switching between single agents has been unsuccessful. The STAR*D study showed that, in earlier stages of treatment resistance, there is little difference in outcome between switching and combination/augmentation unless a patient has shown a partial response to a particular antidepressant medication. In the latter situation, augmenting the effect of the relevant antidepressant
TABLE 1 Some other augmenting agents used in treatment-resistant depression

\begin{tabular}{|lll|}
\hline Augmenting agent & Proposed mechanism & Comment \\
\hline Pindolol & $5-\mathrm{HT}_{1 \mathrm{~A}}$ receptor antagonist & $\begin{array}{l}\text { May speed SSRI onset; ineffective in TRD } \\
\text { in larger trials (Perez 1979) }\end{array}$ \\
\hline Buspirone & $5-\mathrm{HT}_{1 \mathrm{~A}}$ partial agonist & $\begin{array}{l}\text { Used in STAR }{ }^{*} \text { D; no consistent placebo- } \\
\text { controlled data suggesting efficacy in TRD }\end{array}$ \\
\hline Lamotrigine & Glutamate antagonist & $\begin{array}{l}\text { Effective in bipolar depression; uncertain } \\
\text { efficacy in TRD (Barbee 2011) }\end{array}$ \\
\hline S-adenosylmethionine & Methyl donor & $\begin{array}{l}\text { Effective in study of SSRI-resistant } \\
\text { patients (Papakostas 2010) }\end{array}$ \\
\hline Folic acid & Methyl donor & $\begin{array}{l}\text { Ineffective in a recent large trial (Bedson } \\
2014)\end{array}$ \\
\hline Metyrapone & Blocks cortisol synthesis & $\begin{array}{l}\text { Ineffective in a recent large trial (Watson } \\
2014)\end{array}$ \\
\hline
\end{tabular}

SSRI, selective serotonin reuptake inhibitor; TRD, treatment-resistant depression

is slightly better than a switch (Gaynes 2012). Numerous agents have been employed to augment the effects of antidepressant drugs and results of the better established combinations are outlined below. Some others are listed in Table 1.

\section{Antidepressant combinations}

Combinations of antidepressants are widely used in resistant depression, but the evidence for this approach is limited. For example, the addition of mirtazapine to ineffective venlafaxine or SSRI treatment is a popular approach, but the randomised trial evidence rests on one small study (Cowen 2005), although a large UK trial is now in progress (www.isrctn.com/ISRCTN06653773). In the USA, the addition of bupropion to SSRIs or serotonin-noradrenaline reuptake inhibitors (SNRIs) is a common practice, and in the STAR*D study adding bupropion to ineffective citalopram treatment was superior to the addition of buspirone on some secondary outcome measures and was better tolerated (Trivedi 2006).

\section{Augmentation with atypical antipsychotic drugs}

The best evidence for augmentation of ineffective SSRI treatment is for the addition of low-dose atypical antipsychotic drugs (Box 1). In a metaanalysis of trials involving 3500 patients, Spielmans and colleagues (2013) found that, relative to placebo, the addition of drugs such as aripiprazole, quetiapine and risperidone to SSRI treatment was significantly more likely to result in clinical remission $(\mathrm{NNT}=9)$. Olanzapine addition was also of benefit, but the effect was small (NNT = 19).

The main problem in the use of atypical antipsychotics is their tolerability and safety. For example, even at low doses, drugs such as olanzapine and quetiapine cause sedation, weight gain and changes in lipid profile, while 
BOX 1 Use of atypical antipsychotics in antidepressant augmentation

- Doses are lower than those used in the treatment of psychosis (aripiprazole $2.5-10 \mathrm{mg} /$ day; olanzapine $2.5-10 \mathrm{mg} /$ day; quetiapine $50-300 \mathrm{mg}$; risperidone $0.5-2.0 \mathrm{mg} /$ day). 'Start low and go slow'.

- Quetiapine (prolonged release) is the only product formally licensed in the UK for add-on treatment in depression unresponsive to antidepressant medication. In practice, immediate-release quetiapine (in the same range of doses) also appears to be effective.

- Formal studies have usually investigated augmentation of ineffective SSRI treatment by atypical agents. However, atypicals also appear effective in enhancing the effect of other monoamine reuptake blockers, including SNRIs and TCAs.

- Most atypical antipsychotics have not been established to have antidepressant activity as monotherapy in unipolar depression. The exception is quetiapine, which has antidepressant activity at doses of $50 \mathrm{mg} /$ day and above. However, quetiapine is not licensed as a monotherapy in depression.

- When using atypical antipsychotics in depression, the same physical and biochemical monitoring is recommended as for their use in psychosis.

aripiprazole is associated with restlessness and extrapyramidal side-effects. In randomised studies, the discontinuation rate due to adverse effects was significantly greater for atypical antipsychotics than for placebo, with a number needed to harm (NNH) of 17 (Nelson 2009).

Another problem is that randomised trials of the addition of atypical antipsychotics in SSRIresistant depression have been short term - usually a matter of weeks - and longer-term efficacy during continuation treatment has not been established.

Finally, it is not clear whether atypical antipsychotics share the same mechanism of action in their augmentation effects. This raises the possibility that even where augmentation with one atypical is unsuccessful, augmentation with another may yet be effective.

\section{Augmentation with lithium or triiodothyronine}

Lithium is still recommended as an augmentation strategy for resistant depression, but compared with atypical antipsychotic addition, the number of patients in randomised placebo-controlled studies is relatively small (under 250) and effect sizes appear smaller in more recent studies. Another issue is that most of the studies of lithium were carried out in patients resistant to tricyclic antidepressants (TCAs) and there are fewer trials looking at the efficacy of lithium in SSRIrefractory patients. Despite this, a recent metaanalysis concluded that lithium was effective in both SSRI- and TCA-resistant patients, and overall the NNT from the randomised studies was around 5 (Nelson 2014).

An open randomised study compared quetiapine augmentation and quetiapine monotherapy with

lithium augmentation in patients who had failed to respond to antidepressant treatment. Quetiapine was non-inferior to lithium and had a numerical advantage on some secondary outcome measures, especially in augmentation therapy, where a 2.3 point advantage over lithium was seen at 6 weeks on the Montgomery-Åsberg Depression Rating Scale. In this study, the best results for lithium augmentation were obtained when serum lithium levels were at least $0.6 \mathrm{mmol} / \mathrm{L}$ (Bauer 2013).

Triiodothyronine $\left(\mathrm{T}_{3}\right)$ augmentation has been little studied in recent years and, as is the case with lithium, the majority of the data concerns augmentation of TCAs. In the STAR*D study, $\mathrm{T}_{3}$ (25-50 $\mathrm{\mu g} /$ day) was non-significantly superior to lithium augmentation in patients who had failed to respond to two previous antidepressant treatment steps and fewer participants dropped out because of intolerance. However, remission rates for both treatments were low (23\% v. 16\%) (Nierenberg 2006). An important limitation is that longerterm safety and efficacy data are lacking with $\mathrm{T}_{3}$ augmentation.

\section{Augmentation with stimulants}

Particularly in the USA, there is a tradition of adding stimulants such as dexamfetamine and methylphenidate to ineffective antidepressant treatment in patients with depression. Although open studies record positive effects, results from controlled trials are less convincing (Corp 2014). There are also trials using modafinil, whose mode of action is not clearly established but which is licensed to reduce sleepiness in patients with narcolepsy. A meta-analysis of four randomised placebo-controlled trials involving about 550 patients indicated that modafinil augmentation increased remission rates in SSRI-resistant patients, with an NNT of 10 (Goss 2013). Such treatment could be considered for SSRIresistant patients when fatigue and sleepiness are problematic, although bupropion may also be helpful in this situation (Papakostas 2006). Modafinil has, however, been associated with adverse hepatic, cardiac and allergic reactions, so care is needed in its use.

\section{Bipolar depression}

The pharmacological treatment of depression in bipolar disorder has some important differences from that of unipolar depression, with less emphasis on antidepressant medication and more on the use of anticonvulsants such as lamotrigine and atypical antipsychotics such as quetiapine, and the combination of olanzapine and fluoxetine (for a full discussion in Advances, see Vázquez 
2014). Many patients with resistant depression, while not meeting formal diagnostic criteria for bipolar disorder, nevertheless exhibit transient symptoms of hypomania or mixed affective states. These individuals can be understood as having a depressive disorder on the 'bipolar spectrum'. Whether or not depression in such patients is better treated along the lines recommended for bipolar depression is not yet established, but is an important topic for research.

\section{Depressive psychosis}

In a randomised study of patients with depressive psychosis, Wijkstra and colleagues (2010) found that response rates were significantly higher for the combination of venlafaxine ( $375 \mathrm{mg} /$ day) and quetiapine $(600 \mathrm{mg} /$ day) than for venlafaxine alone $(66 \%$ v. $33 \%)$. The combination was also numerically (but not statistically) superior to treatment with imipramine alone (52\% response). A meta-analysis of nine trials confirmed that antidepressant-antipsychotic combination treatment is superior to either treatment alone $(\mathrm{NNT}=7$ v. antidepressant monotherapy; NNT $=5 \mathrm{v}$. antipsychotic monotherapy) (Farahani 2012).

These findings confirm the traditional clinical view that effective treatment of depressive psychosis requires a combination of antidepressant and antipsychotic therapy. When using atypical antipsychotics, it is important to give a dose appropriate for the treatment of psychosis rather than the lower doses commonly used to augment antidepressant treatment in non-psychotic depression. It is also worth noting that TCAs may be more effective than the newer antidepressant drugs in the treatment of depressive psychosis (Wijkstra 2010).

\section{Electroconvulsive therapy}

Electroconvulsive therapy continues to have a place in the treatment of patients with resistant depression, although observational studies suggest that a history of antidepressant resistance may lower the expected response rate $(48 \%$ in antidepressantresistant patients v. $65 \%$ in non-treatmentresistant patients) (Heijnen 2010). Nevertheless, in head-to-head comparisons, the efficacy of ECT is superior to that of antidepressant medication and it can be effective in many antidepressantresistant patients (Birkenhäger 2006). Another issue is that antidepressant resistance may be associated with higher relapse rates post-ECT and it is not clear what the best form of continuation treatment might be. A randomised study involving 200 patients found post-ECT treatment with the combination of lithium and nortriptyline to be as effective as maintenance ECT in sustaining remission in the 6 months following a successful course of treatment. Despite this, just over 50\% of patients in each group relapsed (Kellner 2006), although it should be noted that this may not differ greatly from the high relapse rates following remission achieved after multiple pharmacological treatments (Rush 2006).

\section{Other brain stimulation approaches}

\section{Deep brain stimulation}

There is growing interest in the use of other brain stimulation methods in the treatment of depression, although at present these remain experimental in the UK. The most invasive is deep brain stimulation (DBS), in which stimulatory electrodes are implanted in the neural circuitry believed to underpin chronic depression. The site most commonly stimulated has been the subgenual cingulate cortex, and a meta-analysis of four observational studies (66 participants) showed a 12-month remission rate of $26 \%$ (Berlim 2014). DBS is considered to be less invasive than neurosurgery in that the stimulation can be stopped and there is no lesioning of brain tissue. However, the procedure does carry risks and there is a lack of long-term efficacy and safety data. Furthermore, a US multicentre randomised controlled trial of subgenual cingulate DBS was recently halted when interim analysis failed to show evidence of potential benefit. The neural basis of depression has not been reliably established and presumably may differ between individuals - so deciding the correct electrode placement and stimulation parameters is not straightforward. Therefore further controlled trials are needed to determine the role of DBS in refractory depression.

\section{Vagal nerve stimulation}

Vagal nerve stimulation (VNS) is used for the treatment of refractory depression in the USA, but has very limited availability in the UK. VNS is an established treatment for patients with refractory epilepsy, in whom it was noted coincidentally to improve mood. The onset of antidepressant effect with VNS is slow, over many months. This means that long-term follow-up studies are needed to assess its effectiveness - short-term controlled studies are of limited utility in this respect. Berry and colleagues (2013) carried out a patient-level meta-analysis of six out-patient multicentre trials involving patients with refractory depression which allowed comparison of VNS and treatment as usual (TAU) for up to 96 weeks postimplantation. Response rates in the VNS group at 48 and 96 weeks were $28 \%$ and $32 \%$, whereas 
the corresponding figures for the TAU participants were $12 \%$ and $14 \%$ (at 48 weeks $\mathrm{NNT}=5.5$ ). A caution is that in only one of these studies was there a randomised comparison of VNS and TAU, and this showed only a small and non-significant difference between conditions.

\section{Transcranial magnetic stimulation}

Transcranial magnetic stimulation (TMS) uses a powerful magnetic field to produce current flow in neural tissue. The use of appropriately shaped coils allows reasonably localised stimulation of major cortical regions. TMS has been used for many years in clinical neurophysiology to examine cortical integrity and to localise the cortical substrates of specific neuropsychological functions. Clinically, TMS has been used to relieve depressive states, but there is still uncertainty about the best cortical localisation and the appropriate stimulation parameters. Most studies have employed TMS administered to the left prefrontal cortex, with treatment being given each weekday for about 2 weeks.

In a meta-analysis of trials involving about 1300 patients with depression randomised to either active or sham TMS, Allan and colleagues (2011) found a greater clinical response in patients receiving active compared with sham treatment $(36 \%$ v. $15 \%$; NNT $=9)$. The presence of antidepressant resistance was not a predictor of response. Interpretation of the findings is complicated by the difficulty of providing a convincing sham treatment and the lack of longerterm follow-up data. Also, where TMS has been directly compared with ECT, the latter treatment has proved more effective. A meta-analysis of data from 294 patients found the rate of remission with ECT to be $52 \%$, compared with $34 \%$ for TMS $(\mathrm{NNT}=5.5)($ Berlim 2013).

\section{Experimental pharmacological treatments Ketamine}

Ketamine is a widely used general anaesthetic agent that blocks the $N$-methyl-D-aspartate (NMDA) subtype of glutamate receptor. At subanaesthetic doses, NMDA receptor antagonists produce dissociative and hallucinogenic effects indeed, ketamine has been proposed as a useful pharmacological model of schizophrenia.

Recent controlled studies have shown that ketamine, given intravenously at a sub-anaesthetic dose, produces a striking and rapid remission of depressive symptoms in patients resistant to conventional pharmacotherapy. The improvement in depression begins about $1 \mathrm{~h}$ after ketamine administration, as the dissociative symptoms wane, and can last up to 7 days. A systematic review of controlled studies involving 180 patients with depression found higher rates of clinical response to ketamine than to intravenous saline or intravenous midazolam at 1, 3 and 7 days, with an NNT of 3-5 (McGirr 2014).

The rapid and striking antidepressant effect of ketamine in resistant depression is both clinically and theoretically important. The problem with the use of intravenous ketamine in depression is knowing how to maintain the antidepressant response over the medium and longer term. Other, orally available, glutamatergic agents such as riluzole or memantine do not appear able to maintain the acute antidepressant effect of ketamine (Mathew 2010). A form of intranasal ketamine has been developed that appears effective acutely and that might be more suitable for continued treatment. However, there are concerns about possible ketamine toxicity after repeated use, including bladder changes as well adverse psychological effects such as dependence. The development of tolerance may also be a potential problem. Nevertheless, the antidepressant effect of ketamine has focused attention on the role of glutamate in antidepressant action and might conceivably lead to novel classes of antidepressant drug.

\section{Anti-inflammatory drugs}

There is growing interest in the role of inflammation in the pathophysiology of depression. For example, a significant proportion of people with depression manifest increases in circulating levels of inflammatory cytokines such as interleukin-6 (IL-6) and tumour necrosis factor alpha (TNF- $\alpha$ ). Moreover, administration of exogenous cytokines such as interferon can produce depression in up to $30 \%$ of patients (for a review see Maes 2011). Studies suggest that patients with high levels of inflammatory markers prior to treatment do not do well on SSRIs; however, a recent investigation suggests that they may respond better to nortriptyline (Uher 2014). If confirmed, this would have important implications for the use of inflammatory markers in treatment stratification in depression. Indeed, a recent investigation in antidepressant-resistant patients found that those with high levels $(>5 \mathrm{mg} / \mathrm{L})$ of the inflammatory marker C-reactive protein (CRP) improved following treatment with the TNF- $\alpha$ antagonist infliximab (Raison 2013).

Results from four randomised trials suggest that the cyclooxygenase-2 (COX-2) inhibitor celecoxib potentiated the ability of SSRI treatment to ameliorate depressive symptoms; however, these 
patients were not treatment resistant. In contrast, observational studies suggest that the use of nonsteroidal anti-inflammatory drugs (NSAIDs) (but not aspirin or COX-2 inhibitors) may worsen the outcome of SSRI treatment in depression (Gallagher 2012).

\section{Psychosocial approaches}

Psychosocial factors that may be maintaining a depressive disorder should be identified and remedied as far as possible. Often, relationships within a family can be severely strained by unremitting depression and joint interviews can be helpful. Particularly if patients are not working, it is important to give consideration to activity scheduling. Many patients with resistant depression referred to psychiatrists will have had psychological treatment of some form and it is important to assess whether further psychotherapy - probably of a more 'high-intensity', structured nature - might be appropriate.

The role of the specific psychotherapies in treatment-resistant depression has been relatively little studied in randomised trials. In the STAR*D investigation, cognitive-behavioural therapy (CBT) had a remission rate equivalent to nextstep pharmacological treatments in patients for whom first-line SSRI treatment was ineffective (Thase 2007). A large randomised study found that behavioural activation was more effective than CBT in patients with severe depression (Dimidjian 2006). A study of primary care patients who had failed to respond adequately to antidepressant medication found that CBT (a median of 11 sessions given over 6 months) was markedly superior to TAU in terms of response rate at 6 months on the Beck Depression Inventory (46\% v. 22\%; NNT =4) (Wiles 2013).

As previously mentioned, a major clinical problem for patients who have remitted only following several different treatment attempts is the high risk of relapse over the next year; for the patient, this raises the prospect of effectively having to start over again with even less in the armoury. In a systematic review of 25 randomised trials, Biesheuvel-Leliefeld and colleagues (2015) found that psychological treatments were better than TAU in preventing relapse/recurrence $(\mathrm{NNT}=5)$ and were a little more effective than antidepressants $(\mathrm{NNT}=13)$, although there was considerable heterogeneity in study designs and clinical outcome definitions. The effect was significantly greater when psychological treatment had been used in the acute phase. The best evidence for specific therapies started following remission or in patients with residual symptoms is for CBT and mindfulness-based cognitive therapy (MBCT), especially in individuals with multiply recurrent illness ( $>3$ episodes). In spite of a lack of direct evidence in patients who have previously shown treatment resistance, the high risk of early relapse means that CBT or MBCT should routinely be considered for relapse prevention.

\section{Conclusions}

Depression that persists despite initial treatment is an important problem in psychiatric practice. Particularly where patients show a good adjustment prior to illness, it is worthwhile pursuing pharmacological management in a collaborative and structured way because eventually most patients will achieve remission. Pharmacological treatment should always be combined with psychosocial approaches, which can range from the supportive emotional connection important in all therapeutic relationships to high-intensity structured psychotherapies (Box 2). Psychological treatment seems especially important following remission to try to reduce the high risk of relapse.

Following failure to respond adequately to an initial treatment, switching antidepressant medication is the most straightforward approach, bearing in mind that venlafaxine, amitriptyline and clomipramine are probably slightly more effective than SSRIs, but carry a greater sideeffect burden. The best current evidence for effective treatment of initial non-response is for augmentation of SSRIs with atypical antipsychotic drugs, but the significant adverse effect burden of this approach limits its utility and makes it more of a third-line treatment. Combinations of antidepressants, particularly mirtazapine

\section{BOX 2 Practical suggestions for the management of resistant depression}

- Make a structured, individualised treatment plan

- Carry out regular standardised assessments, e.g. the nine-item Patient Health Questionnaire (PHQ-9), the Beck Depression Inventory (BDI), the Hamilton Rating Scale for Depression (HRSD)

- Plan the 'next-step' treatment from the outset (the current one may well not work)

- Be clear about the timescale and 'do something'

- Combine drug treatments with psychosocial approaches

- Consider electroconvulsive therapy at all stages if symptoms are severe

- Prioritise relapse prevention when recovery occurs, especially adjunctive cognitive-behavioural therapy or mindfulness-based cognitive therapy 


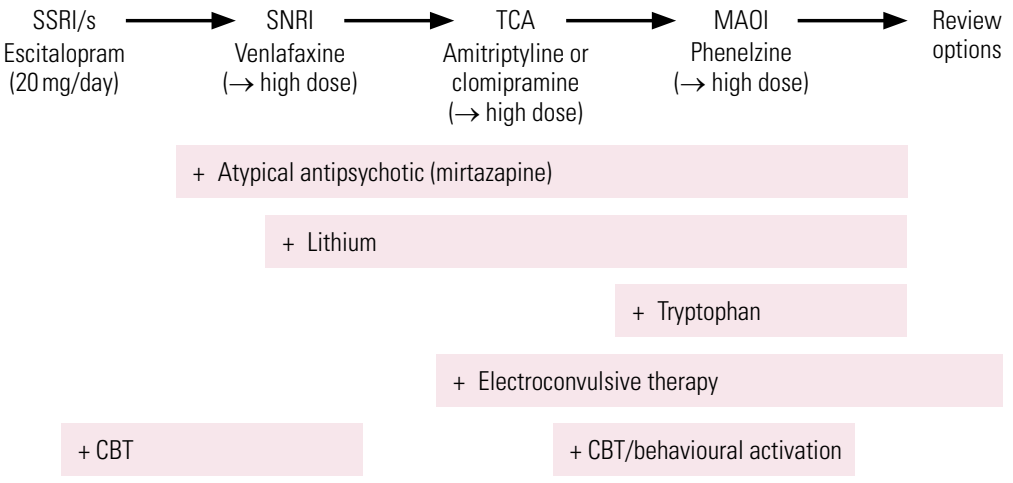

FIG 1

A possible treatment algorithm for depression: adapt to clinical history and gaps in treatment. CBT, cognitive-behavioural therapy; MAOI, monamine oxidase inhibitor; SNRI, serotonin-noradrenaline reuptake inhibitor; SSRI, selective serotonin reuptake inhibitor; TCA, tricyclic antidepressant. High-dose suggestions, if safety and tolerance permit: venlafaxine $300-450 \mathrm{mg} / \mathrm{day}$; amitriptyline and clomipramine $200 \mathrm{mg} /$ day and above; phenelzine $60-90 \mathrm{mg} /$ day.

with SSRIs/SNRIs and bupropion with SSRIs, are widely used alternatives, but lack a strong evidence base. Lithium augmentation is still an important treatment, but its place is now usually after atypical antipsychotics have been tried. The use of ECT is declining, but it remains a uniquely effective treatment in patients who fail to respond to pharmacological measures, or indeed at any point where the need for response is urgent. MAOIs, often combined with lithium, may offer respite for patients not responding to these steps. Figure 1 shows a treatment algorithm summarising the next-step approach.

Whether patients with resistant depression on the 'bipolar spectrum' may do better with treatments such as quetiapine and lamotrigine is currently unclear, but this approach does offer some additional options.

The future pharmacological treatment of resistant depression is likely to feature the use of novel mechanisms, for example glutamatergic agents and anti-inflammatory drugs. Particularly with anti-inflammatory drugs, there seems to be a realistic prospect of finding peripheral biomarkers that will help match patients to treatment. This would be a very important development because even though with currently available treatments most depressive episodes will remit eventually, the time taken to find a suitable therapeutic approach results in much suffering and disability, and may contribute to the high risk of subsequent relapse.

\section{References}

Allan CL, Herrmann LL, Ebmeier KP (2011) Transcranial magnetic stimulation in the management of mood disorders. Neuropsychobiology, 64: 163-9.

MCO answers

1 e 2 b 3 c 4 e 5 e
Anderson IM (2003) Drug treatment of depression: reflections on the evidence. Advances in Psychiatric Treatment, 9: 11-20.
Anderson IM (2013) Pharmacological treatment of unipolar depression. Current Topics in Behavioural Neurosciences, 14: 263-89.

Barbee JG, Thompson TR, Jamhour NJ, et al (2011) A double-blind placebo-controlled trial of lamotrigine as an antidepressant augmentation agent in treatment-refractory unipolar depression. Journal of Clinical Psychiatry, 72: 1405-12.

Bauer M, Pfenning A, Linden M, et al (2009) Efficacy of an algorithmguided treatment compared with treatment as usual: a randomized controlled study of inpatients with depression. Journal of Clinical Psychopharmacology, 29: 327-33.

Bauer M, Dell'Osso L, Kasper S, et al (2013) Extended-release quetiapine fumarate (quetiapine XR) monotherapy and quetiapine XR or lithium as add-on to antidepressants in patients with treatment-resistant major depressive disorder. Journal of Affective Disorders, 151: 209-19.

Bedson E, Bell D, Carr D, et al (2014) Folate Augmentation of Treatment - Evaluation for Depression (FolATED): randomised trial and economic evaluation. Health and Technology Assessments, 18: 1-159.

Bennabi D, Aouizerate B, El-Hage W, et al (2015) Risk factors for treatment resistance in unipolar depression: a systematic review. Journal of Affective Disorders, 171: 137-41.

Berlim MT, Van den Eynde F, Daskalakis ZJ (2013) Efficacy and acceptability of high frequency repetitive transcranial magnetic stimulation (RTMS) versus electroconvulsive therapy (ECT) for major depression: a systematic review and meta-analysis of randomized trials. Depression and Anxiety, 30: 614-23.

Berlim MT, McGirr A, Van den Eynde F, et al (2014) Effectiveness and acceptability of deep brain stimulation (DBS) of the subgenual cingulate cortex for treatment resistant depression: a systematic review and exploratory meta-analysis. Journal of Affective Disorders, 159: 31-8.

Berry SM, Broglio K, Bunker M, et al (2013) A patient-level meta-analysis of studies evaluating vagus nerve stimulation therapy for treatmentresistant depression. Medical Devices (Auckland, NZ), 6: 17-35.

Biesheuvel-Leliefeld KE, Kok GD, Bockting CL, et al (2015) Effectiveness of psychological interventions in preventing recurrence of depressive disorder: meta-analysis and meta-regression. Journal of Affective Disorders, 174: 400-10.

Birkenhäger TK, Van den Broek WW, Moleman P, et al (2006) Outcome of a 4-step treatment algorithm for depressed patients. Journal of Clinical Psychiatry, 67: 1266-71.

Corp SA, Gitlin MJ, Altshuler LL (2014) A review of the use of stimulants and stimulant alternatives in treating bipolar depression and major depressive disorder. Journal of Clinical Psychiatry, 75: 1010-8.

Cowen PJ (2005) New drugs, old problems. Advances in Psychiatric Treatment, 11: 19-27.

Cowen PJ (2011) Has psychopharmacology got a future? British Journal of Psychiatry, 198: 333-5.

Dimidjian S, Hollon SD, Dobson KS, et al (2006) Randomized trial of behavioral activation, cognitive therapy, and antidepressant medication in the acute treatment of adults with major depression. Journal of Consulting and Clinical Psychology, 74: 658-70.

Farahani A, Correll CU (2012) Are antipsychotics or antidepressants needed for psychotic depression? A systematic review and meta-analysis of trials comparing antidepressant or antipsychotic monotherapy with combination treatment. Journal of Clinical Psychiatry, 73: 486-96.

Fekadu A, Wooderson S, Donaldson C, et al (2009) A multidimensional tool to quantify treatment resistance in depression: the Maudsley Staging Method. Journal of Clinical Psychiatry, 70: 177-84.

Gallagher PJ, Castro V, Fava M, et al (2012) Antidepressant response in patients with major depression exposed to NSAIDs: a pharmacovigilance study. American Journal of Psychiatry, 169: 1065-72.

Gaynes BN, Dusetzina SB, Ellis AR, et al (2012) Treating depression after initial treatment failure: directly comparing switch and augmenting strategies in STAR*D. Journal of Clinical Psychopharmacology, 32: 114-9.

Goss AJ, Kaser M, Costafreda SG, et al (2013) Modafinil augmentation therapy in unipolar and bipolar depression: a systematic review and meta-analysis of randomized controlled trials. Journal of Clinical Psychiatry, 74: 1101-7. 
Heijnen WT, Birkenhäger TK, Wierdsma Al, et al (2010) Antidepressant pharmacotherapy failure and response to subsequent electroconvulsive therapy: a meta-analysis. Journal of Clinical Psychopharmacology, 30: $616-9$.

Kellner CH, Knapp RG, Petrides G, et al (2006) Continuation electroconvulsive therapy vs pharmacotherapy for relapse prevention in major depression: a multisite study from the Consortium for Research in Electroconvulsive Therapy (CORE). Archives of General Psychiatry, 63: 1337-44.

Maes M (2011) Depression is an inflammatory disease, but cell-mediated immune activation is the key component of depression. Progress in Neuro-Psychopharmacology and Biological Psychiatry, 35: 664-75

Mathew SJ, Murrough JW, Collins KA, et al (2010) Riluzole for relapse prevention following intravenous ketamine in treatment-resistant depression: a pilot randomized, placebo-controlled continuation trial. International Journal of Neuropsychopharmacology, 13: 71-82.

McGirr A, Berlim MT, Bond DJ, et al (2014) A systematic review and meta-analysis of randomised, double-blind placebo-controlled trials of ketamine in the rapid treatment of major depressive episodes. Psychological Medicine, 10: 1-12.

National Institute for Health and Care Excellence (2009) Depression: The Treatment and Management of Depression in Adults (NICE Clinical Guideline 90). NICE.

Nelson JC, Papakostas GI (2009) Atypical antipsychotic augmentation in major depressive disorder: a meta-analysis of placebo-controlled randomized trials. American Journal of Psychiatry, 166: 980-91.

Nelson JC, Baumann P, Delucchi K, et al (2014) A systematic review and meta-analysis of lithium augmentation in tricyclic and second generation antidepressants in major depression. Journal of Affective Disorders, 156 : 269-75.

Nierenberg A, Fava M, Trivedi M, et al (2006) Comparison of lithium and T3 augmentation following two failed medication treatments for depression: a STAR* ${ }^{*}$ report. American Journal of Psychiatry, 163: 1519-30.

Papakostas GL, Nutt DJ, Hallett LA, et al (2006) Resolution of sleepiness and fatigue in major depressive disorder: a comparison of bupropion and the selective serotonin reuptake inhibitors. Biological Psychiatry, 60: $1350-5$

Papakostas G, Fava M, Thase ME (2008) Treatment of SSRI-resistant depression: a meta-analysis comparing within- versus across-class switches. Biological Psychiatry, 63: 699-704.
Papakostas GI, Mischoulon D, Shuyu I, et al (2010) S-adenosyl methionine (SAMe) augmentation of serotonin reuptake inhibitors for antidepressant nonresponders with major depressive disorder: a double-blind, randomized clinical trial. American Journal of Psychiatry, 167: 942-8.

Perez V, Soler J, Puigdemont D, et al (1979) A double-blind, randomized, placebo-controlled trial of pindolol augmentation in depressive patients resistant to serotonin reuptake inhibitors. Archives of General Psychiatry, 56: 375-9.

Raison CL, Rutherford RE, Woolwine BJ, et al (2013) A randomized controlled trial of the tumour necrosis factor antagonist infliximab for treatment-resistant depression: the role of baseline inflammatory biomarkers. Archives of General Psychiatry, 70: 31-41.

Rush AJ, Trivedi MH, Wisniewski SR, et al (2006) Acute and longer-term outcomes in depressed outpatients requiring one or several treatment steps: a STAR ${ }^{*}$ D report. American Journal of Psychiatry, 163: 1905-17.

Spielmans GI, Berman MI, Linardatos E, et al (2013) Adjunctive atypical antipsychotic treatment for major depressive disorder: a meta-analysis of depression, quality of life, and safety outcomes. PLoS Medicine, 10(3): e1001403.

Thase ME, Friedman ES, Biggs MM, et al (2007) Cognitive therapy versus medication in augmentation and switch strategies as second-step treatments: a STAR ${ }^{*}$ D report. American Journal of Psychiatry, 164: 739-52.

Trivedi MH, Fava M, Wisniewski SR, et al (2006) Medication augmentation after the failure of SSRls for depression. New England Journal of Medicine, 354: 1243-52.

Uher R, Tansey KE, Maier W, et al (2014) An inflammatory biomarker as a differential predictor of outcome of depression treatment with escitalopram and nortriptyline. American Journal of Psychiatry, 171: 1278-86.

Vázquez GH, Tondo L, Undurraga J, et al (2014) Pharmacological treatment of bipolar depression. Advances in Psychiatric Treatment, 20: 193-201.

Watson S, Anderson IM, Apekey TA, et al (2014) Antiglucocorticoid augmentation of antidepressants in depression: the ADD study. Journal of Psychopharmacology, 28(8) (suppl): A38

Wijkstra J, Burger H, Van den Broek WW, et al (2010) Treatment of unipolar psychotic depression: a randomized, double-blind study comparing imipramine, venlafaxine, and venlafaxine plus quetiapine. Acta Psychiatrica Scandinavica, 121: 190-200

Wiles N, Thomas L, Abel A, et al (2013) Cognitive behavioural therapy as an adjunct to pharmacotherapy for primary care based patients with treatment resistant depression: results of the CoBalT randomised controlled trial. Lancet, 381: 375-84.

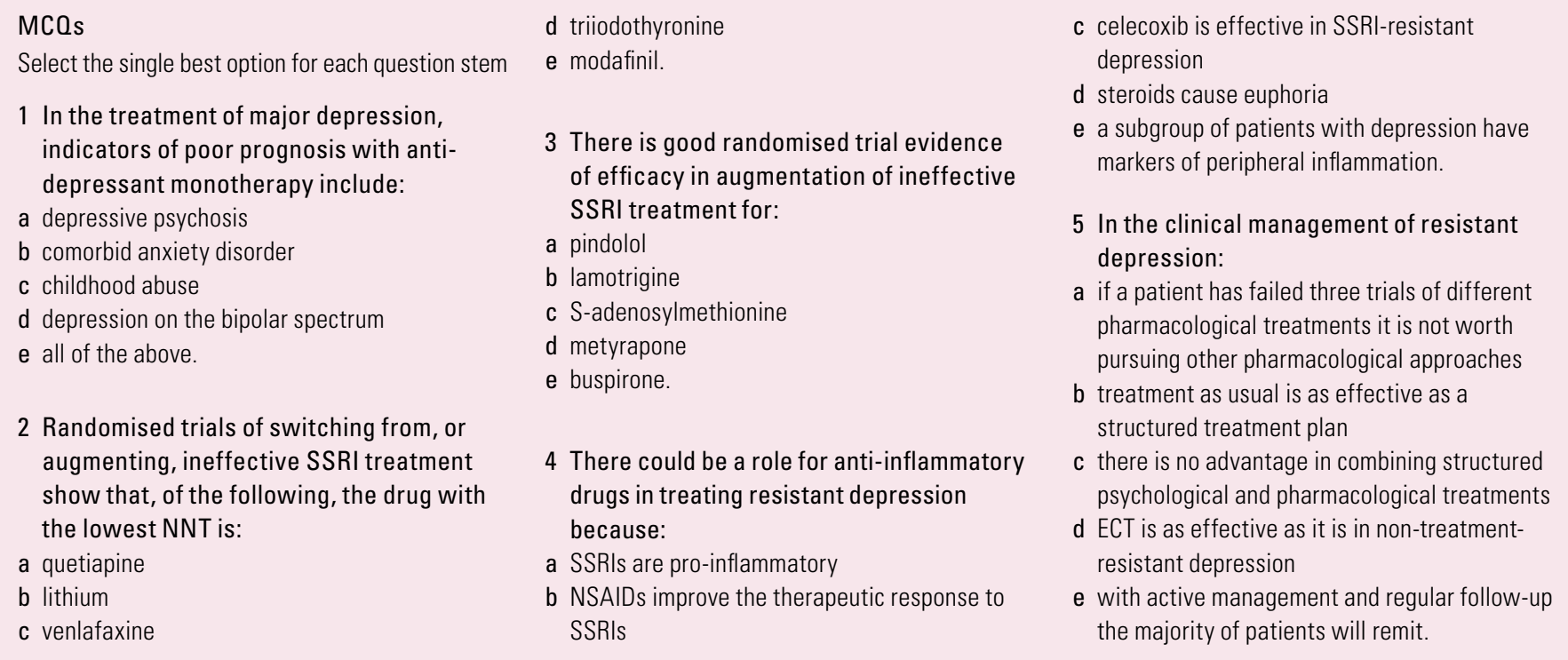

